\title{
Review of the current literature regarding cardiac adverse events following COVID-19 vaccination
}

\author{
Tufan Çınar ${ }^{*}$ (D), Mert IIlker Hayıroğlu² (D), Vedat Çiçek ${ }^{1}$, \\ Murat Selçuk' ${ }^{1}$, Samet Yavuz ${ }^{1}$, Ahmet Lütfullah Orhan ${ }^{1}$
}

\section{INTRODUCTION}

The severe acute respiratory syndrome coronavirus 2 (SARSCoV-2), which was responsible for coronavirus disease 2019 (COVID-19) infection, was discovered in Wuhan, China, in December $2019^{1}$. Since then, the disease has spread globally, resulting in a pandemic. Because there is no specific antiviral treatment for COVID-19 disease, vaccination seems to appear the most effective vehicle for controlling the infection. Until now, many vaccines have been developed and approved for immediate use by the health authorities. Two types of messenger RNA (mRNA)based COVID-19 vaccines, namely, BNT162b2 mRNA (PfizerBioNTech, NY) and mRNA-1273 (Moderna, Cambridge, MA), have been administered in hundreds of millions of doses since they have received provisional Food and Drug Administration (FDA) approval in the United States in December 2020․ Janssen Ad26.COV2.S (Johnson and Johnson, New Brunswick, NJ) and The ChAdOx1 [Oxford/AstraZeneca (AZD1222)] were recombinant types of vaccines, in which replication-deficient human adenovirus type 26 vector was used to transfer the virus ${ }^{1}$. Although side effects from these vaccines are generally mild and transient, there has been an upsurge of cases with cardiac adverse events reported after COVID-19 vaccination. As a result, the objective of this review was to assess all cardiovascular adverse events reported following COVID-19 immunization, as well as the likely mechanisms behind them.

\section{METHODS}

We searched the database of PubMed, Embase, and Cochrane for all possible cardiac adverse events reported after COVID-19 vaccination using the following search inputs until September 13, 2021: "COVID-19 vaccine-induced acute myocarditis," "COVID-19 vaccine-induced acute perimyocarditis," "COVID-19 vaccine-induced acute myocardial infarction," "COVID-19 vaccine-induced ST elevation myocardial infarction," and "COVID-19 vaccine-induced acute coronary syndrome." Only papers written in English were included in this review. Additionally, following a review of the references in the relevant publications, any further papers were collected. Our review was restricted to only cardiac adverse events reported after COVID-19 vaccination. In total, 68 relevant cases were found in the literature. Of them, 61 cases were diagnosed with acute myocarditis (AM), one case with acute perimyocarditis, five cases with acute myocardial infarction (AMI), and one case with Kounis syndrome after COVID19 vaccination.

\section{Vaccination types}

Table 1 describes the vaccine types, symptoms onset, and COVID-19 polymerase chain reaction (PCR) positivity of all published cases. The majority of AM patients who suffered cardiac adverse events after receiving COVID-19 vaccination had previously been immunized with mRNA-based vaccines. [In total $=65$ cases, 35 of them with BNT $162 \mathrm{~b} 2$ (Pfizer) and 30 of them with mRNA-1273 SARS-CoV-2 (Moderna $)^{2-15}$.] Only three cases had a history of vaccination with adenovirus vector origin [two with Covishield (AZD1222) and one with Janssen Ad.26.COV2.S (Johnson and Johnson) ${ }^{2}$.] Almost all of the AM cases (60/61) were diagnosed following the injection of vaccinations made with mRNA technology, and the majority of them developed

\footnotetext{
${ }^{1}$ Health Sciences University, Sultan Abdulhamid Han Training and Research Hospital, Department of Cardiology - Istanbul, Turkey.

${ }^{2}$ Health Sciences University, Dr. Siyami Ersek Training and Research Hospital, Department of Cardiology - Istanbul, Turkey.

*Corresponding author: drtufancinar@gmail.com

Conflicts of interest: the authors declare there is no conflicts of interest. Funding: none.

Received on September 21, 2021. Accepted on September 26, 2021.
} 
Table 1. Vaccine types, symptoms onset, and COVID-19 PCR positivity of all published cases.

\begin{tabular}{|c|c|c|c|c|c|c|}
\hline & $\begin{array}{l}\text { Vaccine } \\
\text { types }\end{array}$ & $\begin{array}{l}\text { Presentation } \\
\text { after the second } \\
\text { dose vaccine (\%) }\end{array}$ & $\begin{array}{l}\text { History of } \\
\text { COVID-19? } \\
(\%)\end{array}$ & $\begin{array}{l}\text { Is the patient } \\
\text { COVID-19 } \\
\text { polymerase chain } \\
\text { reaction positive? }\end{array}$ & $\begin{array}{l}\text { Does the } \\
\text { patient have a } \\
\text { nucleocapsid } \\
\text { antibody? }\end{array}$ & $\begin{array}{l}\text { Time between } \\
\text { last vaccination } \\
\text { and symptom } \\
\text { onset, days }\end{array}$ \\
\hline $\begin{array}{l}\text { Case } \\
\text { series }^{2}\end{array}$ & $\begin{array}{c}5 \text { BNT162b2 } \\
\text { (Pfizer); } \\
1 \text { mRNA-1273 } \\
\text { (Moderna); } 1 \\
\text { J\&J }\end{array}$ & 71 & 14 & $\begin{array}{l}\text { 6/7 patients were } \\
\text { tested, all were } \\
\text { negative }\end{array}$ & $\begin{array}{l}\text { 4/7 patients tested, } \\
\text { all were negative }\end{array}$ & $3(2-7)$ \\
\hline $\begin{array}{l}\text { Case } \\
\text { series }^{3}\end{array}$ & $\begin{array}{l}5 \text { BNT 162b2 } \\
\text { (Pfizer); } \\
3 \text { mRNA1273 } \\
\text { (Moderna) }\end{array}$ & 88 & 25 & All were negative & NA & $3(1-4)$ \\
\hline $\begin{array}{l}\text { Case } \\
\text { series }^{4}\end{array}$ & $\begin{array}{l}\text { BNT 162b2 } \\
\text { (Pfizer) }\end{array}$ & 83 & No & All were negative & All were negative & $2.5(1-16)$ \\
\hline $\begin{array}{l}\text { Case } \\
\text { series }^{5}\end{array}$ & $\begin{array}{l}2 \text { BNT 162b2 } \\
\text { (Pfizer), } \\
2 \text { mRNA1273 } \\
\text { (Moderna) }\end{array}$ & 100 & No & Negative & NA & $2.5(1-5)$ \\
\hline $\begin{array}{l}\text { Case } \\
\text { series }^{6}\end{array}$ & $\begin{array}{c}7 \text { BNT 162b2 } \\
\text { (Pfizer), } \\
16 \text { mRNA1273 } \\
\text { (Moderna) }\end{array}$ & 87 & 13 & $\begin{array}{l}\text { 19/23 were tested, all } \\
\text { were negative }\end{array}$ & NA & $2(1-4)$ \\
\hline Case $^{7}$ & $\begin{array}{l}\text { BNT62b2 } \\
\text { (Pfizer) }\end{array}$ & 100 & 100 & Negative & $\% 100$ & 3 \\
\hline Case $^{8}$ & $\begin{array}{l}\text { BNT162b2 } \\
\text { (Pfizer) }\end{array}$ & 100 & No & Negative & Negative & 1 \\
\hline Case $^{9}$ & $\begin{array}{l}\text { BNT162b2 } \\
\text { (Pfizer) }\end{array}$ & 100 & No & Negative & Negative & 3 \\
\hline Case $^{10}$ & $\begin{array}{l}\text { mRNA-1273 } \\
\text { (Moderna) }\end{array}$ & 100 & No & Negative & NA & 4 \\
\hline Case $^{11}$ & $\begin{array}{l}\text { mRNA-1273 } \\
\text { (Moderna) }\end{array}$ & 100 & No & Negative & Negative & 1 \\
\hline Case $^{12}$ & $\begin{array}{l}\text { BNT62b2 } \\
\text { (Pfizer) }\end{array}$ & 100 & No & Negative & Negative & 1 \\
\hline Case $^{13}$ & $\begin{array}{c}\text { BNT62b2 } \\
\text { (Pfizer) }\end{array}$ & 100 & No & Negative & Negative & 3 \\
\hline Case $^{14}$ & $\begin{array}{l}\text { mRNA-1273 } \\
\text { (Moderna) }\end{array}$ & 100 & No & Negative & Negative & 1 \\
\hline $\begin{array}{l}\text { Case } \\
\text { series }^{15}\end{array}$ & $\begin{array}{l}\text { BNT62b2 } \\
\text { (Pfizer) }\end{array}$ & 100 & No & Negative & Negative & $12 \mathrm{~h}-3$ \\
\hline Case $^{16}$ & $\begin{array}{l}\text { BNT62b2 } \\
\text { (Pfizer) }\end{array}$ & No* & No & Negative & Negative & $1 \mathrm{~h}$ \\
\hline Case $^{17}$ & $\begin{array}{l}\text { Covishield } \\
\text { (Azd1222) }\end{array}$ & $\mathrm{No}^{*}$ & No & Negative & Negative & 2 \\
\hline Case $^{18}$ & $\begin{array}{l}\text { mRNA-1273 } \\
\text { (Moderna) }\end{array}$ & $\mathrm{No}^{*}$ & No & Negative & Negative & 1 \\
\hline $\begin{array}{l}\text { Case } \\
\text { series }^{19}\end{array}$ & $\begin{array}{c}\text { mRNA-1273 } \\
\text { (Moderna) }\end{array}$ & $\mathrm{No}^{*}$ & No & Negative & Negative & $1-5$ \\
\hline Case 20 & $\begin{array}{c}\text { Azd1222 } \\
\text { (Oxford } \\
\text { University and } \\
\text { AstraZeneca) }\end{array}$ & $\mathrm{No}^{*}$ & No & Negative & Negative & $2 \mathrm{~h}$ \\
\hline
\end{tabular}

*Presentation after the first dose vaccine. NA: not applicable. 
symptoms 1-3 days after the second dose of immunization $(57 / 61)^{2-15}$. In contrast to AM cases, the majority of AMI cases (4/5) emerged after the first dose of mRNA-based vaccination was administered ${ }^{16-19}$. Only one case of Kounis syndrome had been reported in the literature, and this allergic response occurred $2 \mathrm{~h}$ after the first dose of Covishield (AZD1222) vaccination ${ }^{20}$. Interestingly, the COVID-19 PCR test was negative in all cases.

\section{Baseline clinical characteristics, electrocardiographic findings, and laboratory findings}

Table 2 summarizes the baseline characteristics, presenting symptoms, electrocardiography, and laboratory results in all published cases. Patients who were diagnosed with AM were relatively younger and almost all of them were male. By contrast, AMI cases were older. The common complaint in all patients was chest pain. Electrocardiography findings in AM cases ranged from no ischemic changes to ST elevation, PR depression, and nonspecific ST changes ${ }^{2-15}$. Remarkably, patients who presented with AMI following immunization had ST elevation only in inferior leads ${ }^{16-20}$. Troponin levels were measured in all patients who developed a cardiac event after vaccination. In all of them, it was reported above the reference range. The data on brain natriuretic peptide (BNP) levels were shared in very few cases $^{2,5,10-12,13-15}$. On the other hand, C-reactive protein (CRP) levels were elevated in all reported cases. Contrary to COVID19 infection, lymphopenia was not detected in most patients with post-vaccine cardiac events.

\section{Imaging findings}

Table 3 displays the imaging data, in-hospital treatment, and outcomes of all cases. Echocardiography was performed in most cases since it was in the diagnostic algorithm of diseases such as AM and AMI. Left ventricle wall motion defect was observed in all patients with AMI, whereas AM patients had findings in the spectrum from preserved left ventricular ejection fraction (LVEF) without segmental abnormalities to global hypokinesia and low LVEF$^{2-6,8-11,13-16}$. Cardiac magnetic resonance imaging was performed in almost all AM cases, which demonstrated a subepicardial late gadolinium enhancement and myocardial edema compatible with $\mathrm{AM}^{2-15}$. Although it was considered the gold standard for the diagnosis of AM, the endomyocardial biopsy was not performed on any patients.

Table 2. Baseline characteristics, presenting symptoms, electrocardiography, and laboratory findings of all published cases.

\begin{tabular}{|c|c|c|c|c|c|}
\hline & $\begin{array}{l}\text { Age, } \\
\text { gender }\end{array}$ & $\begin{array}{l}\text { Presenting } \\
\text { symptoms }\end{array}$ & Diagnosis & $\begin{array}{l}\text { Electrocardiographic } \\
\text { (ECG) findings }\end{array}$ & $\begin{array}{l}\text { Lab } \\
\text { findings }\end{array}$ \\
\hline $\begin{array}{l}\text { Case } \\
\text { series }^{2}\end{array}$ & $\begin{array}{c}24(19-30), \\
\text { all cases } \\
\text { were male }\end{array}$ & $\begin{array}{c}\text { Chest pain was } \\
\text { present in all cases, } \\
42 \% \text { had nonspecific } \\
\text { symptoms }\end{array}$ & AM & $\begin{array}{c}4 \text { patients had ST elevations, } \\
1 \text { patient had nonspecific ST/T } \\
\text { changes }\end{array}$ & $\begin{array}{c}\text { Lymphopenia: - } \\
\text { CRP: elevated in } 71 \% \\
\text { Troponin: elevated in all cases } \\
\text { BNP: elevated in } 50 \%\end{array}$ \\
\hline $\begin{array}{l}\text { Case } \\
\text { series }^{3}\end{array}$ & $\begin{array}{c}29(21-56), \\
\text { all cases } \\
\text { were male }\end{array}$ & $\begin{array}{l}\text { Chest pain was } \\
\text { present in all cases, } \\
63 \% \text { had nonspecific } \\
\text { symptoms }\end{array}$ & AM & $\begin{array}{l}6 \text { patients had ST elevation, } 1 \\
\text { patient had peaked T waves, } \\
\text { one patient had normal ECG }\end{array}$ & $\begin{array}{c}\text { Lymphopenia: - } \\
\text { CRP: elevated in } 88 \% \\
\text { Troponin: elevated in all } \\
\text { cases }\end{array}$ \\
\hline $\begin{array}{l}\text { Case } \\
\text { series }^{4}\end{array}$ & $\begin{array}{l}22(16-45), \\
\text { all cases } \\
\text { were male }\end{array}$ & $\begin{array}{c}\text { Chest pain was } \\
\text { present in all cases, } \\
33 \% \text { had nonspecific } \\
\text { symptoms }\end{array}$ & AM & All cases had ST elevations & $\begin{array}{l}\text { Lymphopenia: - } \\
\text { CRP: elevated in all cases } \\
\text { Troponin: elevated in all } \\
\text { cases }\end{array}$ \\
\hline $\begin{array}{l}\text { Case } \\
\text { series }^{5}\end{array}$ & $\begin{array}{l}30(23-70), \\
75 \% \text { of } \\
\text { cases were } \\
\text { male }\end{array}$ & $\begin{array}{l}\text { Chest pain was } \\
\text { present in all cases, } \\
33 \% \text { had nonspecific } \\
\text { symptoms }\end{array}$ & AM & $\begin{array}{l}\text { All cases had ST elevation, } \\
\text { two cases had PR depression }\end{array}$ & $\begin{array}{c}\text { Lymphopenia: - } \\
\text { CRP: elevated in all cases } \\
\text { Troponin: elevated in all } \\
\text { cases } \\
\text { BNP: elevated in } 50 \%\end{array}$ \\
\hline $\begin{array}{l}\text { Case } \\
\text { series }^{6}\end{array}$ & $\begin{array}{l}25(20-51), \\
\text { all cases } \\
\text { were male }\end{array}$ & $\begin{array}{l}\text { Chest pain was } \\
\text { present in all cases }\end{array}$ & AM & $\begin{array}{l}\text { 19/23 cases had ST } \\
\text { elevations, T-wave inversions, } \\
\text { and nonspecific ST changes }\end{array}$ & $\begin{array}{c}\text { Lymphopenia: - } \\
\text { CRP: NA } \\
\text { Troponin: elevated in all cases }\end{array}$ \\
\hline Case $^{7}$ & 56, Male & Chest pain & AM & ST elevation & $\begin{array}{l}\text { Lymphopenia: - } \\
\text { CRP: elevated } \\
\text { Troponin: elevated }\end{array}$ \\
\hline
\end{tabular}


Table 2. Continuation.

\begin{tabular}{|c|c|c|c|c|c|}
\hline & $\begin{array}{l}\text { Age, } \\
\text { gender }\end{array}$ & $\begin{array}{l}\text { Presenting } \\
\text { symptoms }\end{array}$ & Diagnosis & $\begin{array}{l}\text { Electrocardiographic } \\
\text { (ECG) findings }\end{array}$ & $\begin{array}{l}\text { Lab } \\
\text { findings }\end{array}$ \\
\hline Case $^{8}$ & 39, Male & $\begin{array}{l}\text { Chest pain, myalgia, } \\
\text { fatigue, fever }\end{array}$ & AM & ST elevation & $\begin{array}{l}\text { Lymphopenia: - } \\
\text { CRP: NA } \\
\text { Troponin: elevated }\end{array}$ \\
\hline Case $^{9}$ & 30, Male & $\begin{array}{l}\text { Chest pain, myalgia, } \\
\text { fatigue, fever }\end{array}$ & AM & ST elevation & $\begin{array}{l}\text { Lymphopenia: - } \\
\text { CRP: elevated } \\
\text { Troponin: elevated }\end{array}$ \\
\hline Case $^{10}$ & 24, Male & $\begin{array}{l}\text { Chest pain, myalgia, } \\
\text { fatigue, fever }\end{array}$ & AM & No ischemic changes & $\begin{array}{l}\text { Lymphopenia: - } \\
\text { CRP: elevated } \\
\text { Troponin: elevated } \\
\text { BNP: normal }\end{array}$ \\
\hline Case $^{11}$ & 52 , Male & $\begin{array}{l}\text { Chest pain, myalgia, } \\
\text { fatigue, fever }\end{array}$ & AM & $\begin{array}{l}\text { Incomplete right bundle } \\
\text { branch block and left axis } \\
\text { deviation }\end{array}$ & $\begin{array}{l}\text { Lymphopenia: - } \\
\text { CRP: elevated } \\
\text { Troponin: elevated } \\
\text { BNP: elevated }\end{array}$ \\
\hline Case $^{12}$ & 66, Male & $\begin{array}{l}\text { Chest pain, myalgia, } \\
\text { fatigue, fever }\end{array}$ & AM & ST elevation & $\begin{array}{l}\text { Lymphopenia: - } \\
\text { CRP: NA } \\
\text { Troponin: elevated }\end{array}$ \\
\hline Case $^{13}$ & 24, Male & $\begin{array}{l}\text { Chest pain, myalgia, } \\
\text { fatigue, fever }\end{array}$ & AM & No ischemic findings & $\begin{array}{l}\text { Lymphopenia: - } \\
\text { CRP: elevated } \\
\text { Troponin: elevated } \\
\text { BNP: elevated }\end{array}$ \\
\hline Case $^{14}$ & 34, Male & $\begin{array}{l}\text { Chest pain, myalgia, } \\
\text { fatigue, fever }\end{array}$ & AM & $\begin{array}{l}\text { Lateral PR depression and } \\
\text { ST elevation mirrored in aVR } \\
\text { with PR elevation and ST } \\
\text { depression }\end{array}$ & $\begin{array}{l}\text { Lymphopenia: - } \\
\text { CRP: elevated } \\
\text { Troponin: elevated } \\
\text { BNP: elevated }\end{array}$ \\
\hline $\begin{array}{l}\text { Case } \\
\text { series }^{15}\end{array}$ & $\begin{array}{l}15 \text { and } 22, \\
\text { all cases } \\
\text { were male }\end{array}$ & $\begin{array}{l}\text { Chest pain, myalgia, } \\
\text { fatigue, fever }\end{array}$ & $\begin{array}{l}\text { AM and acute } \\
\text { myopericarditis }\end{array}$ & $\begin{array}{l}\text { J-point elevation in the lateral } \\
\text { leads with slightly widened } \\
\text { QRS complexes and no } \\
\text { ischemic findings }\end{array}$ & $\begin{array}{l}\text { Lymphopenia: - } \\
\text { CRP: elevated } \\
\text { Troponin: elevated } \\
\text { BNP: elevated }\end{array}$ \\
\hline Case $^{16}$ & 86, Male & $\begin{array}{l}\text { Chest pain, myalgia, } \\
\text { fatigue, fever }\end{array}$ & STEMI & ST elevation inferior wall & $\begin{array}{l}\text { Lymphopenia: - } \\
\text { CRP: NA } \\
\text { Troponin: NA } \\
\text { BNP: NA }\end{array}$ \\
\hline Case $^{17}$ & 63, Male & $\begin{array}{l}\text { Chest pain, myalgia, } \\
\text { fatigue, fever }\end{array}$ & STEMI & $\begin{array}{c}\text { ST elevation in leads } \| \text { and III } \\
\text { and aVF }\end{array}$ & $\begin{array}{l}\text { Lymphopenia: NA } \\
\text { CRP: NA } \\
\text { Troponin: elevated }\end{array}$ \\
\hline Case $^{18}$ & 96, Female & $\begin{array}{l}\text { Chest pain, myalgia, } \\
\text { fatigue, fever }\end{array}$ & STEMI & $\begin{array}{l}\text { ST-segment elevation in the } \\
\text { anterior and inferior leads }\end{array}$ & $\begin{array}{l}\text { Lymphopenia: NA } \\
\text { CRP: NA } \\
\text { Troponin: elevated }\end{array}$ \\
\hline $\begin{array}{l}\text { Case } \\
\text { series }^{19}\end{array}$ & $\begin{array}{l}42 \text { and } 68, \\
50 \% \text { of } \\
\text { cases were } \\
\text { male }\end{array}$ & Chest pain & STEMI and AMI & $\begin{array}{c}\text { ST elevation in leads } \| \text { and III } \\
\text { and aVF }\end{array}$ & $\begin{array}{l}\text { Lymphopenia: NA } \\
\text { CRP: NA } \\
\text { Troponin: elevated }\end{array}$ \\
\hline Case $^{20}$ & 62, Female & Chest pain & $\begin{array}{l}\text { Kounis } \\
\text { syndrome }\end{array}$ & $\begin{array}{c}\text { ST elevation in inferior leads } \\
\text { (II, III, and AVF) and reciprocal } \\
\text { ST segment depression in } \\
\text { lead I and AVL }\end{array}$ & $\begin{array}{l}\text { Lymphopenia: NA } \\
\text { CRP: NA } \\
\text { Troponin: elevated }\end{array}$ \\
\hline
\end{tabular}

AM: acute myocarditis; CRP: C-reactive protein; BNP: brain natriuretic peptide; STEMI: ST elevation myocardial infarction; AMI: acute myocardial infarction; NA: not applicable. 
Table 3. Imaging findings, in-hospital treatment, and outcomes of all published cases.

\begin{tabular}{|c|c|c|c|c|}
\hline & $\begin{array}{l}\text { Echocardiographic } \\
\text { findings (\%) }\end{array}$ & Cardiac MRI findings & $\begin{array}{c}\text { Median } \\
\text { hospitalization, } \\
\text { days (range) }\end{array}$ & $\begin{array}{c}\text { In-hospital treatment } \\
(\%)\end{array}$ \\
\hline $\begin{array}{l}\text { Case } \\
\text { series }^{2}\end{array}$ & $\begin{array}{c}\text { Abnormal in } 57 \text { [mild } \\
\text { hypokinesia in 3, } 1 \text { reduced } \\
\text { LVEF, one mild LV enlargement], } \\
\text { normal in } 43 .\end{array}$ & $\begin{array}{c}\text { All cases had LGE, } \\
\text { one with wall motion } \\
\text { abnormality, three with } \\
\text { myocardial edema in T2 }\end{array}$ & $3(2-4)$ & $\begin{array}{l}43 \text { with NSAIDs, } 43 \\
\text { with colchicine, } 43 \text { with } \\
\text { famotidine, } 14 \text { with } \\
\text { steroids }\end{array}$ \\
\hline $\begin{array}{l}\text { Case } \\
\text { series }^{3}\end{array}$ & $\begin{array}{l}\text { All cases had motion } \\
\text { abnormality with regional or } \\
\text { generalized hypokinesia }\end{array}$ & $\begin{array}{l}\text { All cases had LGE, six with } \\
\text { myocardial edema }\end{array}$ & $\begin{array}{l}\text { All cases were } \\
\text { reported as stable }\end{array}$ & $\begin{array}{l}38 \text { with NSAIDs, } 25 \\
\text { with colchicine, } 13 \text { with } \\
\text { steroids }\end{array}$ \\
\hline $\begin{array}{l}\text { Case } \\
\text { series }^{4}\end{array}$ & $\begin{array}{c}\text { 2/6 with hypokinetic segments } \\
\text { but preserved LVEF, } 4 / 6 \text { had } \\
\text { normal LVEF }\end{array}$ & $\begin{array}{c}\text { All cases had mild } \\
\text { subepicardial edema and } \\
\text { LGE }\end{array}$ & $6(4-8)$ & $\begin{array}{l}100 \text { with NSAIDs and } \\
\text { colchicine }\end{array}$ \\
\hline $\begin{array}{l}\text { Case } \\
\text { series }^{5}\end{array}$ & $\begin{array}{l}\text { One patient with LVEF } 40 \text {, the } \\
\text { others had normal LVEF }\end{array}$ & $\begin{array}{c}\text { All cases had LGE, } \\
\text { increased T1 and T2 } \\
\text { intensity }\end{array}$ & $3(2-4)$ & $\begin{array}{c}50 \text { with NSAIDs, } 75 \\
\text { with colchicine, } 25 \text { with } \\
\text { steroid }\end{array}$ \\
\hline $\begin{array}{l}\text { Case } \\
\text { series }^{6}\end{array}$ & 4/23 cases had LVEF $<50$ & $\begin{array}{c}\text { All cases had subepicardial } \\
\text { LGE or focal myocardial } \\
\text { edema }\end{array}$ & NA & NA \\
\hline Case $^{7}$ & NA & $\begin{array}{l}\text { LGE and myocardial edema } \\
\text { in T2 imaging }\end{array}$ & 7 & NA \\
\hline Case $^{8}$ & Normal LVEF & $\begin{array}{l}\text { Subepicardial } \\
\text { enhancement }\end{array}$ & 6 & $\begin{array}{l}\text { Anti-inflammatory } \\
\text { medications }\end{array}$ \\
\hline Case $^{9}$ & $\begin{array}{l}\text { Abnormal wall motion } \\
\text { abnormality and mild pericardial } \\
\text { effusion }\end{array}$ & $\begin{array}{l}\text { Subepicardial LGE of the } \\
\text { myocardium }\end{array}$ & 7 & $\begin{array}{l}\text { Beta-blocker, } \\
\text { acetylsalicylic acid, } \\
\text { steroid }\end{array}$ \\
\hline Case $^{10}$ & Normal LVEF & $\begin{array}{l}\text { Patchy mid-myocardial and } \\
\text { epicardial LGE with edema }\end{array}$ & NA & Beta-blocker \\
\hline Case $^{11}$ & $\begin{array}{l}\text { No wall motion abnormalities, } \\
\text { LVEF was preserved }\end{array}$ & $\begin{array}{c}\text { Mild myocardial and } \\
\text { subepicardial linear and } \\
\text { nodular LGE and mild } \\
\text { hypokinesia }\end{array}$ & 4 & $\begin{array}{l}\text { ACE inhibitor, beta- } \\
\text { blocker }\end{array}$ \\
\hline Case $^{12}$ & Reduced LVEF of 44 & $\begin{array}{l}\text { Edema on T2 sequences } \\
\text { and subepicardial } \\
\text { enhancement in the lateral } \\
\text { mediastinal region }\end{array}$ & NA & NA \\
\hline Case $^{13}$ & Normal LVEF & $\begin{array}{c}\text { Subepicardial } \\
\text { enhancement involving the } \\
\text { lateral wall }\end{array}$ & NA & NA \\
\hline Case $^{14}$ & $\begin{array}{l}\text { Reduced LVEF of } 43 \text { without } \\
\text { pericardial effusion }\end{array}$ & $\begin{array}{c}\text { Subepicardial LGE in } \\
\text { the anterolateral and } \\
\text { inferolateral segments, as } \\
\text { well as patchy myocardial } \\
\text { edema on T2 }\end{array}$ & 5 & $\begin{array}{l}\text { High-dose aspirin, } \\
\text { colchicine, ACE } \\
\text { inhibitor, beta-blocker }\end{array}$ \\
\hline $\begin{array}{l}\text { Case } \\
\text { series }^{15}\end{array}$ & Normal LVEF & NA & 2 & $\begin{array}{l}\text { Aspirin, NSAIDs, and } \\
\text { colchicine }\end{array}$ \\
\hline Case $^{16}$ & NA & NA & Not survived & $\begin{array}{l}\text { Balloon angioplasty } \\
\text { and glycoprotein llb/ } \\
\text { Illa receptor inhibitor } \\
\text { (eptifibatide) }\end{array}$ \\
\hline
\end{tabular}


Table 3. Continuation.

\begin{tabular}{|c|c|c|c|c|}
\hline & $\begin{array}{l}\text { Echocardiographic } \\
\text { findings }(\%)\end{array}$ & Cardiac MRI findings & $\begin{array}{c}\text { Median } \\
\text { hospitalization, } \\
\text { days (range) }\end{array}$ & $\begin{array}{c}\text { In-hospital treatment } \\
(\%)\end{array}$ \\
\hline Case $^{17}$ & $\begin{array}{l}\text { Inferior wall hypokinesia with } \\
\text { LVEF of } 50\end{array}$ & NA & 5 & $\begin{array}{l}\text { Thrombolysed } \\
\text { with } 1.5 \text { million IU } \\
\text { streptokinase, and } \\
\text { anti-platelets and anti- } \\
\text { anginal drugs }\end{array}$ \\
\hline Case $^{18}$ & $\begin{array}{c}\text { Anterior and inferior wall } \\
\text { hypokinesia with LVEF of } 35 \%\end{array}$ & NA & 3 & Heparin \\
\hline $\begin{array}{l}\text { Case } \\
\text { series }^{19}\end{array}$ & $\begin{array}{l}\text { LVEF of } 50 \% \text { and hypokinesia } \\
\text { of the anterolateral and } \\
\text { inferolateral walls and LVEF of } \\
60 \% \text {, with hypokinetic inferior } \\
\text { and inferolateral walls }\end{array}$ & NA & $2-7$ & $\mathrm{PCl}$ \\
\hline Case $^{20}$ & $\begin{array}{l}\text { Inferior wall motion abnormality } \\
\text { and preserved LVEF }\end{array}$ & NA & 3 & $\mathrm{PCl}$ \\
\hline
\end{tabular}

MRI: magnetic resonance imaging; LVEF: left ventricle ejection fraction; LGE: late gadolinium enhancement; NA: not applicable; NSAIDs: nonsteroidal anti-inflammatory drugs; PCl: percutaneous coronary intervention.

\section{In-hospital treatment and outcomes}

No case of acute fulminant myocarditis was reported after COVID-19 vaccination. Most reported AM cases were hospitalized for 3-5 days on average, and all of them were discharged uneventfully ${ }^{2-5,7-9,11,14,15}$. In the treatment of AM, high-dose aspirin, colchicine, beta-blockers, and steroids were most preferred $^{1-5,12,14}$. In addition to anti-ischemic and anti-aggregant therapy, the primary percutaneous coronary intervention was performed in patients presenting with AMI ${ }^{16-20}$. All AMI cases were discharged uneventfully, except the 86-year-old male patient who did not survive during the in-hospital course ${ }^{16}$.

\section{DISCUSSION}

AM is generally regarded as an uncommon adverse effect following vaccination. According to reports, the majority of previously documented post-vaccine AM cases were subclinical and were discovered by routine pre- and post-vaccine troponin level assessments ${ }^{21}$. However, in our review, all the cases documented following COVID-19 immunization were symptomatic. This implies that asymptomatic individuals might not be identified, and as a result, cardiac events following immunization might be significantly greater than predicted.

Although the causes of AM due to COVID-19 vaccinations are not well understood, several potential pathophysiological explanations have been proposed. It has been considered that in some people with genetic vulnerability, the immunological response to mRNA-based COVID-19 vaccines may be uncontrollable, resulting in the activation of an abnormal innate and acquired immune response ${ }^{22}$. Also, both dendritic cells and Toll-like receptor-expressing cells subjected to mRNA may still be able to produce cytokines in certain people, albeit this may be significantly decreased when exposed to mRNA with nucleoside alterations as opposed to unmodified $\mathrm{RNA}^{22}$. As a result, the immune system may recognize the mRNA as an antigen, leading to hyperactivation of the inflammatory and immunologic pathways, which may have a role in the occurrence of AM in certain people as part of a systemic response ${ }^{22}$.

During vaccination, an allergic reaction may develop, which can be classified as a vaccine-related adverse effect. It is always difficult to determine whether a response is caused by the vaccination or by other causes. Adjuvants are usually included in the vaccines to enhance stability, solubility, and absorption, which can result in IgE-mediated anaphylactic responses following immunization. This might be one explanation for AMI following the COVID-19 vaccination. The fact that all published AMI cases had their complaints started within a short time after the initial dosage of vaccination supports this hypothesis. Another potential AMI cause, as proposed by Warkentin et al., is vaccine-induced prothrombotic immune thrombocytopenia, which is similar to heparin-induced thrombocytopenia and leads to thrombotic manifestation ${ }^{23}$. 


\section{Future perspective}

The number of documented cases supports the "very uncommon" interpretation of vaccine-related cardiac side effects despite the fact that hundreds of millions of COVID-19 vaccinations have been administered globally. It was also clearly demonstrated that the majority of the patients with cardiac adverse events demonstrated full recovery in terms of both symptoms and imaging. Moreover, it must be highlighted that since there has been no causative link between COVID-19 vaccinations and cardiac events, the effectiveness of the COVID-19 vaccination far exceeds some possible drawbacks. Consequently, more research on AM, AMI, and other cardiac events before and after COVID-19 vaccination will enrich the literature about the long-term effects of the vaccination and determining the incidence rate.

\section{AUTHORS' CONTRIBUTIONS}

TÇ: Conceptualization, Formal Analysis, Writing - original draft, Writing - review \& editing. MIH: Conceptualization, Formal Analysis, Writing - review \& editing. ALO: Supervision, Formal Analysis, Writing - review \& editing. VÇ: Data curation, Funding acquisition, Resources, Writing - review \& editing. MS: Data curation, Funding acquisition, Resources, Writing review \& editing. SY: Funding acquisition, Resources, Writing - review \& editing.

\section{REFERENCES}

1. Hippisley-Cox J, Coupland CA, Mehta N, Keogh RH, Diaz-Ordaz K, Khunti K, et al. Risk prediction of covid-19 related death and hospital admission in adults after covid-19 vaccination: national prospective cohort study. BMJ. 2021;374:n2244. https://doi.org/10.1136/bmj.n2244

2. Rosner CM, Genovese L, Tehrani BN, Atkins M, Bakhshi H, Chaudhri $S$, et al. Myocarditis temporally associated with COVID-19 vaccination. Circulation. 2021;144(6):502-5. https:// doi.org/10.1161/CIRCULATIONAHA.121.055891

3. Larson KF, Ammirati E, Adler ED, Cooper LT Jr, Hong KN, Saponara G, Couri D, Cereda A, Procopio A, Cavalotti C, Oliva F, Sanna T, Ciconte VA, Onyango G, Holmes DR, Borgeson DD. Myocarditis After BNT162b2 and mRNA-1273 Vaccination. Circulation. 2021;144(6):506-8. https://doi.org/10.1161/ CIRCULATIONAHA. 121.055913

4. Abu Mouch S, Roguin A, Hellou E, Ishai A, Shoshan U, Mahamid $\mathrm{L}$, et al. Myocarditis following COVID-19 mRNA vaccination. Vaccine. 2021;39(29):3790-3. https://doi.org/10.1016/j. vaccine.2021.05.087

5. Kim HW, Jenista ER, Wendell DC, Azevedo CF, Campbell MJ, Darty SN, Parker MA, Kim RJ. Patients with acute myocarditis following mRNA COVID-19 vaccination. JAMA Cardiol. 2021;6(10):1196-201. https://doi.org/10.1001/ jamacardio.2021.2828

6. Montgomery J, Ryan M, Engler R, Hoffman D, McClenathan $B$, Collins $L$, et al. Myocarditis following immunization with mRNA COVID-19 vaccines in members of the us military. JAMA Cardiol. 2021;6(10):1202-6. https://doi.org/10.1001/ jamacardio.2021.2833

7. Ammirati E, Cavalotti C, Milazzo A, Pedrotti P, Soriano F, Schroeder JW, et al. Temporal relation between second dose BNT162b2 mRNA Covid-19 vaccine and cardiac involvement in a patient with previous SARS-COV-2 infection. Int J Cardiol Heart Vasc. 2021:100778. https://doi.org/10.1016/j.jjcha.2021.100778

8. García JB, Ortega PP, Fernández JAB, León AC, Burgos LR, Dorta EC. Acute myocarditis after administration of the BNT162b2 vaccine against COVID-19. Rev Esp Cardiol (Engl Ed). 2021;74(9):812-4. https://doi.org/10.1016/j.rec.2021.04.005
9. D'Angelo T, Cattafi A, Carerj ML, Booz C, Ascenti G, Cicero $G$, et al. Myocarditis after SARS-CoV-2 vaccination: a vaccineinduced reaction? Can J Cardiol. 2021:S0828-282X(21)00286-5. https://doi.org/10.1016/j.cjca.2021.05.010

10. Albert E, Aurigemma G, Saucedo J, Gerson DS. Myocarditis following COVID-19 vaccination. Radiol Case Rep. 2021;16(8):2142-5. https://doi.org/10.1016/j.radcr.2021.05.033

11. Muthukumar A, Narasimhan M, Li QZ, Mahimainathan L, Hitto I, Fuda $F$, et al. In-depth evaluation of a case of presumed myocarditis after the second dose of COVID-19 mRNA vaccine. Circulation. 2021;144(6):487-98. https://doi.org/10.1161/ CIRCULATIONAHA. 121.056038

12. Gautam N, Saluja P, Fudim M, Jambhekar K, Pandey T, Al'Aref S. A late presentation of COVID-19 vaccine-induced myocarditis. Cureus. 2021;13(9):e17890. https://doi.org/10.7759/cureus.17890

13. Singh $B$, Kaur $P$, Cedeno L, Brahimi T, Patel P, Virk H, Shamoon F, Bikkina M. COVID-19 mRNA Vaccine and Myocarditis. Eur J Case Rep Intern Med. 2021;8(7):002681. https://doi. org/10.12890/2021_002681

14. Williams CB, Choi Jl, Hosseini F, Roberts J, Ramanathan K, Ong K. Acute Myocarditis Following mRNA-1273 SARS-CoV-2 Vaccination. CJC Open. 2021 Jul 14. https://doi.org/10.1016/j. cjco.2021.07.008

15. Hudson B, Mantooth R, DeLaney M. Myocarditis and pericarditis after vaccination for COVID-19. J Am Coll Emerg Physicians Open. 2021;2(4):e12498. https://doi.org/10.1002/emp2.12498

16. Tajstra M, Jaroszewicz J, Gąsior M. Acute coronary tree thrombosis after vaccination for COVID-19. JACC CardiovasC Interv. 2021;14(9):e103-4. https://doi.org/10.1016/j. jcin.2021.03.003

17. Chatterjee S, Ojha UK, Vardhan B, Tiwari A. Myocardial infarction after COVID-19 vaccination-casual or causal? Diabetes Metab Syndr. 2021;15(3):1055-6. https://doi.org/10.1016/j.dsx.2021.04.006

18. Boivin Z, Martin J. Untimely myocardial infarction or COVID-19 vaccine side effect. Cureus. 2021;13(3):e13651. https://doi. org/10.7759/cureus. 13651 
19. Sung JG, Sobieszczyk PS, Bhatt DL. Acute myocardial infarction within 24 hours after COVID-19 vaccination. Am J Cardiol. 2021;156:129-31. https://doi.org/10.1016/j. amjcard.2021.06.047

20. Maadarani $O$, Bitar Z, Elzoueiry M, Nader M, Abdelfatah M, Zaalouk T, et al. Myocardial infarction post COVID-19 vaccine - coincidence, Kounis syndrome or other explanation - time will tell. JRSM Open. 2021;12(8):20542704211025259. https://doi.org/10.1177/20542704211025259

21. Cassimatis DC, Atwood JE, Engler RM, Linz PE, Grabenstein JD, Vernalis MN. Smallpox vaccination and myopericarditis: a clinical review. J Am Coll Cardiol. 2004;43(9):1503-10. https:// doi.org/10.1016/j.jacc.2003.11.053

22. Karikó K, Buckstein M, Ni H, Weissman D. Suppression of RNA recognition by Toll-like receptors: the impact of nucleoside modification and the evolutionary origin of RNA Immunity. 2005;23(2):165-75. https://doi.org/10.1016/j. immuni.2005.06.008

23. Warkentin TE, Greinacher A. Spontaneous HIT syndrome: knee replacement, infection, and parallels with vaccineinduced immune thrombotic thrombocytopenia. Thromb Res. 2021;204:40-51. https://doi.org/10.1016/j. thromres.2021.05.018

\section{ERRATUM}

https://doi.org/10.1590/1806-9282.20210940ERRATUM

In the manuscript "Review of the current literature regarding cardiac adverse events following COVID-19 vaccination", DOI: 10.1590/1806-9282.20210940, published in the Rev Assoc Med Bras. 2021;67(12):1751-1757, on page 1751:

\section{Where it reads:}

Mert İlker Hayıroçlu

It should read:

Mert İlker Hayıroğlu 